\title{
Study on Blind-guiding Robot Based on STM32 Embedded Systems Changgeng $\mathrm{Yu}^{1, \mathrm{a}^{*}}$, Suyun $\mathrm{Li}^{1, \mathrm{~b},{ }^{*}}$, Qiang $\mathrm{Li}^{1, \mathrm{a}}$ \\ ${ }^{1}$ School of Mechanical and Electronic Engineering, Hezhou University, No.18, xihuan Road, Hezhou,China \\ ayuchanggen66@163.com, ${ }^{b}$ syxwx@126.com
}

\begin{abstract}
Keywords: Blind-guiding Robot; Avoiding Obstruction; Image Recognition; STM32 Embedded Systems
\end{abstract}

\begin{abstract}
The blind guiding robot is problems that solve blind users navigate safely. The scheme adopts the four-wheels vehicles as the main body of the robot, it detects the environment information by the STM32 embedded systems, the many kinds of sensors, and fusion technology was used with different location of the infrared sensors, ultrasonic sensor acquisition to the local environment of information fusion, blind road tracking by visual sensor at the same time, so as to improve the decision-making, planning, reaction of intelligent system of quickness and accuracy; With the method of image identification, add to identify traffic lights to guide system module, identify a zebra crossing and traffic lights, achieve the goal of safe travel for the blind. Using multi-sensor fusion technology, combined with STM32 embedded system can precisely control the car motion trail. It has achieved the purpose of helping blind people travelling safely.
\end{abstract}

\section{Introduction}

World Health Organization estimated that approximately 45 million people worldwide are living with some form of blind ${ }^{[1]}$, while the total number of blind people in China accounted for about $1 / 5$ of the number of blind people in the world, about 19 million to 45 million per year rate of increase ${ }^{[2]}$. For the blind, the world's color is dark; there is a great inconvenience to their lives, study and work. Especially in terms of walking, unpredictable terrain and obstacles for the blind is very dangerous. How can you make the blind walk safety is an important and meaningful topic ${ }^{[3-4]}$.

With growing maturity and development of robot technology, people demand for robots is no longer merely content with the industrial automation production, and put more attention to assist works with disabilities ${ }^{[5]}$, intelligent robots is the development trend of the robot, the development and application of all kinds of control algorithm also provides a more intelligent way of implementation, this topic for the blind to the urgent needs of the independent safety walk, designed a model based on the STM32 seeing-eye robot control system. This system uses a multi-sensor information fusion technology, different position of infrared sensors and ultrasonic sensors to collect information to the local environment comprehensive, while eliminating sensor may exist between the redundant information, reduce the uncertainty, thus improve the intelligent system of decision-making, planning, quickness and accuracy of response. This scheme can be applied to the field of robotics ${ }^{[6]}$.

\section{System Overview}

The trolley system as the main advantage of STM32 microcontroller in order to control the car. Cars including the infrared sensors, ultrasonic sensor and visual sensor, through infrared sensors tracking at the same time using the vision sensor 1 aided tracking and identification of a zebra crossing, ultrasonic sensors used in the small car for obstacle avoidance, finally, the vision sensor. Identification of traffic lights, the use of multi-sensor fusion technology makes the car more intelligent, humane, to help blind people travel safely ${ }^{[7-9]}$. The working principle diagram as shown in Fig. 1. 
First, the system is initialized, press the Start button on the car blind use infrared sensors tracking forward, travel distance by ultrasonic sensors for obstacle detection, timely correct the direction of the robot forward, so as to avoid obstacles, the whole process of car travel by classical PID algorithm; Color sensor to identify the intersection traffic light colors; Ensure the safety of guide the blind by road. Due to road traffic lights installation position is higher, the Angle of vision sensors limit into consideration, so in this system USES two visual sensor, were installed in different positions of the machine, to acquire the information of traffic lights and road surface images at the same time, the recognition of traffic light and image processing with DSP algorithm. The workflow chart is shown in Fig. 2.

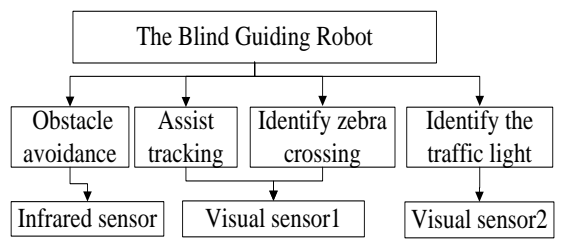

Figure 1. Diagram of the working principle

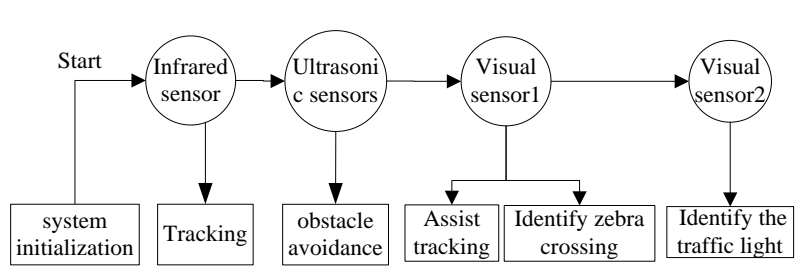

Figure 2. Workflow chart

\section{Hardware Design}

Microcontroller. Single Chip Micyocosystem part of the circuit diagram of main control chip is STM32F103C8T6, mainly includes the reset circuit, clock circuit, MCU input power filter circuit. STM32F103C8T6 is shown in Fig. 3.

Power Supply. The design of the power supply is mainly to the STM32 power supply and other power supply circuit, STM32 chips working voltage of $3.3 \mathrm{~V}$, the voltage by LM1117 3.3. Other circuit working voltage is $5 \mathrm{v}$, can use a $9 \mathrm{v}$ dc after LM7805 converted to $5 \mathrm{v}$. That part of the circuit as shown in Figure 4 and 5, respectively. Circuit inductance L300 ripple wave in order to reduce the input voltage, C301 and C303 for large capacitance, used for filtering processing. But composition of capacitors and inductors, so if you have any high frequency noise here, it's impedance will increase, so beside the capacitance value of capacitance in parallel with a small ceramic capacitor, the large capacitance at low frequency, small capacitance high-frequency, better able to filter out the noise in the power supply.

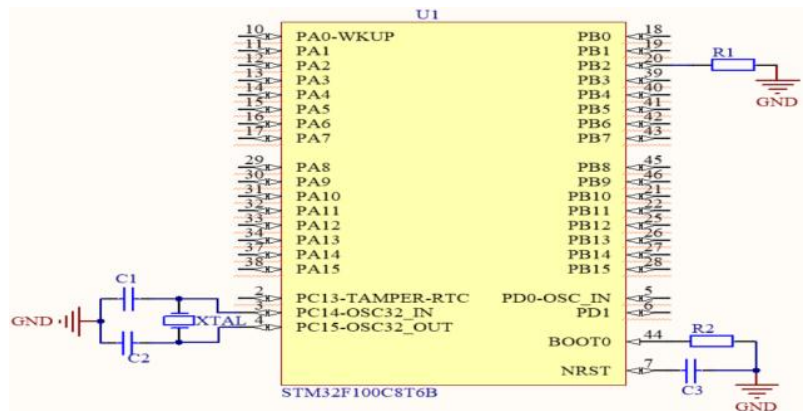

Figure 3. Microcontroller

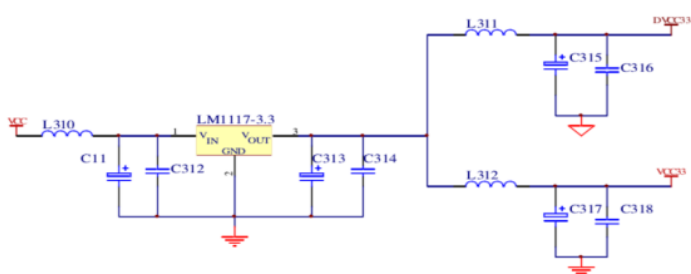

Figure 4. 3.3 voltage converter module

Motor Modular. Motor drive circuit adopted L298N integrated chip module, there are four road output, can be used to control motor rotation, the part of the circuit diagram as shown in Fig. 6 .

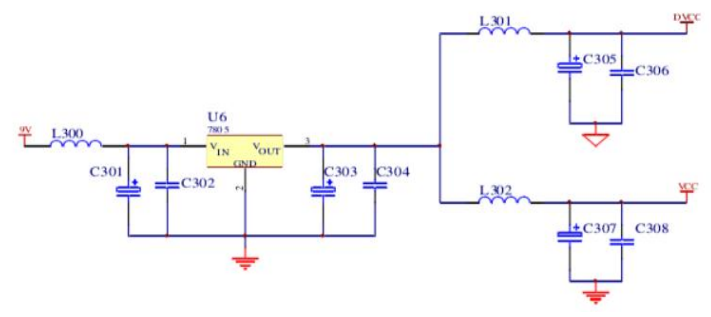

Figure 5. voltage converter module

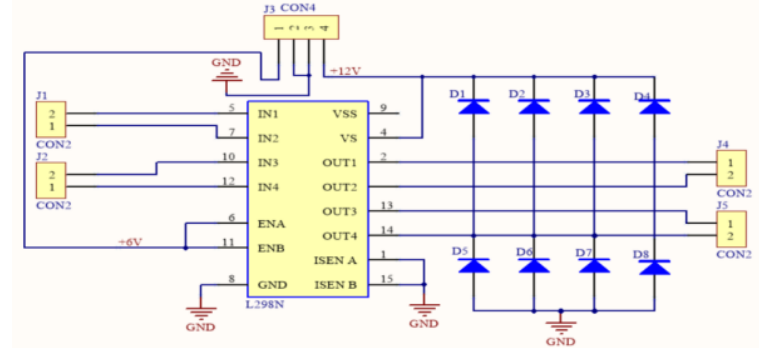

Figure 6. Motor Modular 


\section{Software Design}

Flowchart for Main Program. This summary introduces the overall structure of software system, using the STM32 embedded system, mainly to complete the system initialization, the processing of ultrasonic ranging, infrared sensor ranging, motor drive, wireless receive instruction and so on. When receives the external commands, execute the corresponding instruction program, such as not to receive instruction, program is run obstacle detection, such as detected no obstacles ahead, marching to the target point; When sensors detect obstacles ahead, obstacle avoidance processing service subroutine, to implement obstacle avoidance, wireless data transceiver final; the program main program flow chart shown in Fig. 7.

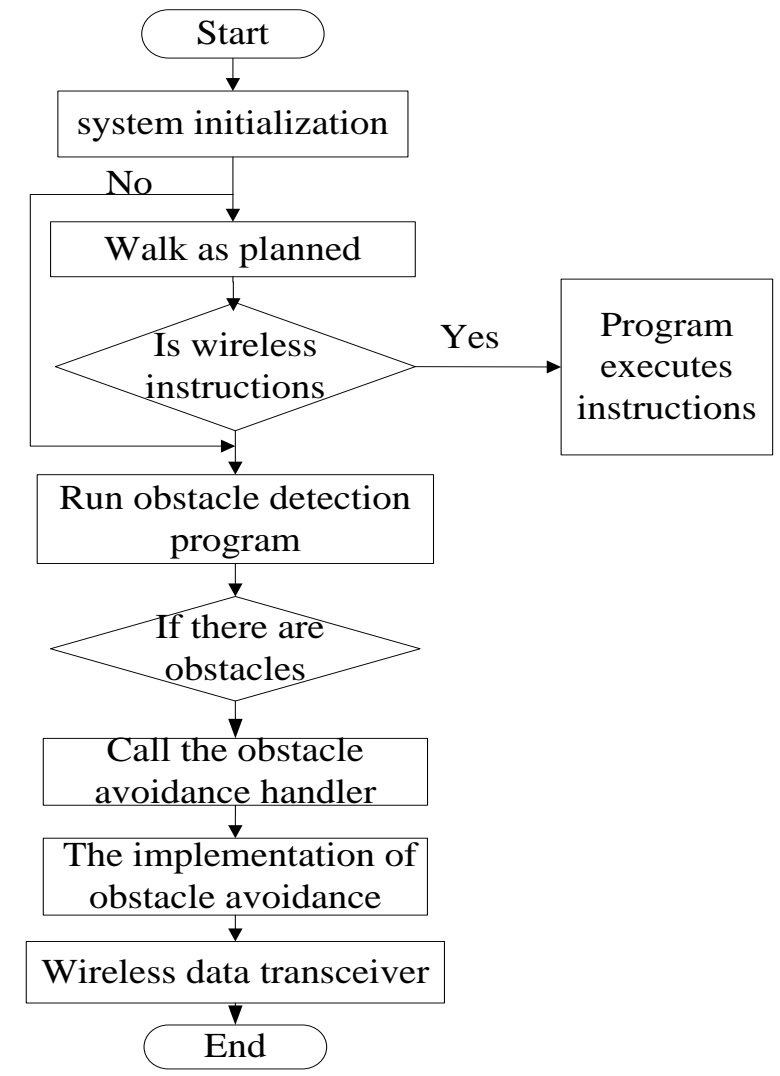

Figure 7. Flowchart for main program

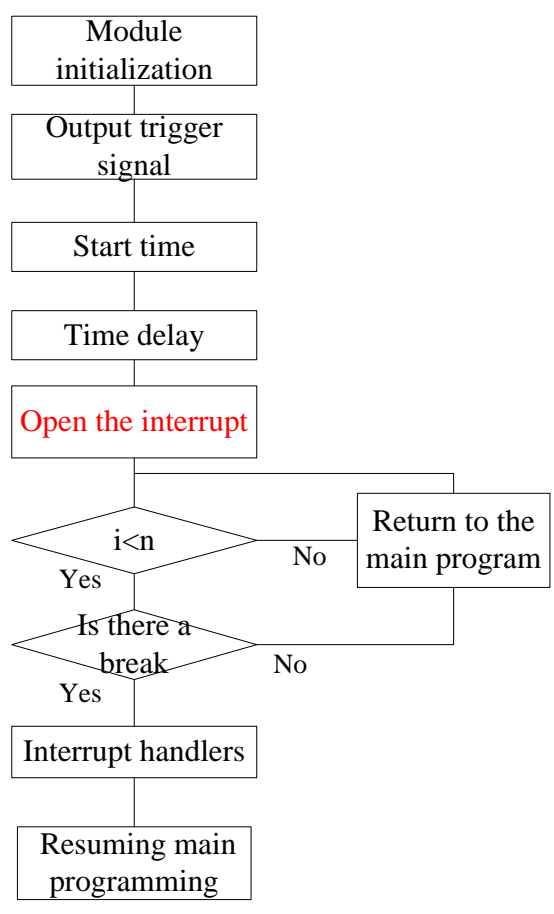

Figure 8. Flowchart for Ultrasonic sensor Module

Flowchart for Obstacle Detection Module. Is the main purpose of this module software design: first, by using the method of delay by the main control chip of an I/O port high level trigger signals are sent to the ultrasonic ranging module, start counting at the same time, in order to prevent the "blind spots" in the range and use the program to the input delay interrupt for a period of time delay, time delay after the completion of the opening of echo signal processing. Interrupt, if any, before the completion of the count, then go to the corresponding interrupt service routine, the obstacle distance is calculated. If there is no this interruption in the process of counting, it returns information accessible in front of the main program to process.

Infrared Sensor Module. Infrared sensor is to use objects to infrared electric switch the infrared emission produced by the presence of strong and weak and detecting position. Photoelectric switch converts input current signal output, light receiver by determining the received light intensity for tracking. 
Under normal condition is not detected at the sensor output as a high level, at the sensor outputs the low level is detected. In practical measurement, the photoelectric switch is connected I/O mouth falling edge set to trigger the interrupt, when the main controller to detect the object caused by interruption, you can determine what is photoelectric switch to detect all the way by which to object, thus for tracking.

Motor Driver Module. This module is mainly for motor PWM control, use a timer TIM4 two output channels for the PWM output of the motor, using the PB10, PB11 as enabling port and a motor driver chip enable port, through adjusting the two port output to control motor and reversing.

Wireless Receiving Module. System USES wireless module for wireless receiver, use master controller USART interfaces and communication module, USART work in two-way traffic, first receive data input (RX) and configured to use to send data output pin (TX), then adopts the model of interrupt receiving data. Using wireless camera to monitor the traffic light and recognition, at the same time through the PC software can constantly monitor the procession to seeing-eye robot.

\section{Prototype and Experiments}

Based on the Keil uVision4 software development, the use of a PC by wireless wifi module sends a command to the STM32 controller STM32 controller respectively control the ultrasonic sensor, motor controller, camera module, and infrared sensors ${ }^{[10]}$; Using the STM32 controller to control the motor controller to control the motor of the car to the car, system debugging block diagram as shown in fig. 9.

In system debugging, used the STM32 embedded control board, including PC and STM32 panel communication using wireless wifi module, the communication advantages including: radio coverage range, transmission speed, can meet the demand of individual and social informatization, debug software, use the upper machine through wireless wifi module sends a command to the STM32 controller to control the corresponding I/O port on the STM32 control board, so as to control the corresponding $\mathrm{I} / \mathrm{O}$ ports on the motor, reach the purpose of controlling the motor car, complete guide function. Seeing-eye robot physical diagram as shown in fig. 10.

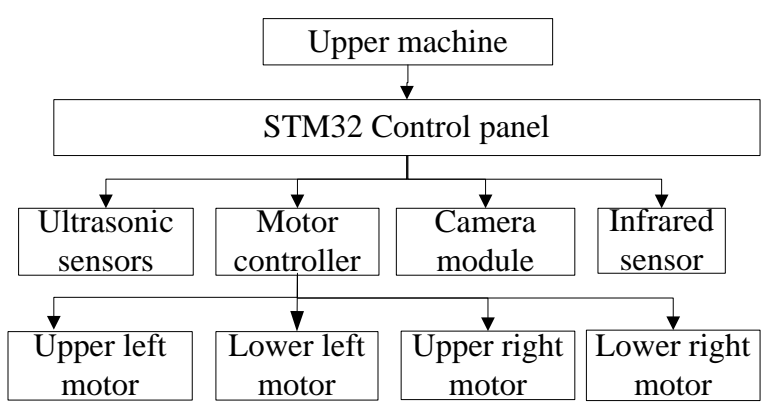

Figure 9. Diagram of the system debugging

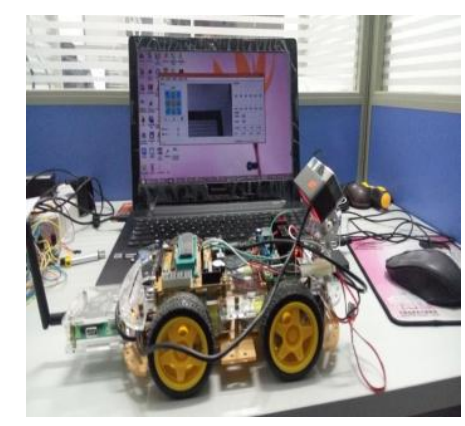

Figure 10. Robot real figure

Debugging of video image monitoring, first open the PC software debugging, and then a channel blind robot power supply, and then search wireless "hot spot" of the robot, the robot and the upper unit into a wireless local area network (LAN), set up epistatic machine and the robot's IP address and port number, if the connection is successful, you can see the video image. Video image is shown in figure 11.

Seeing-eye robot part module diagram is shown in figure 12. PWM signals generated by using the controller combined with corresponding sensor control speed and direction of the robot, debug software through the upper machine can control the robot movement. 


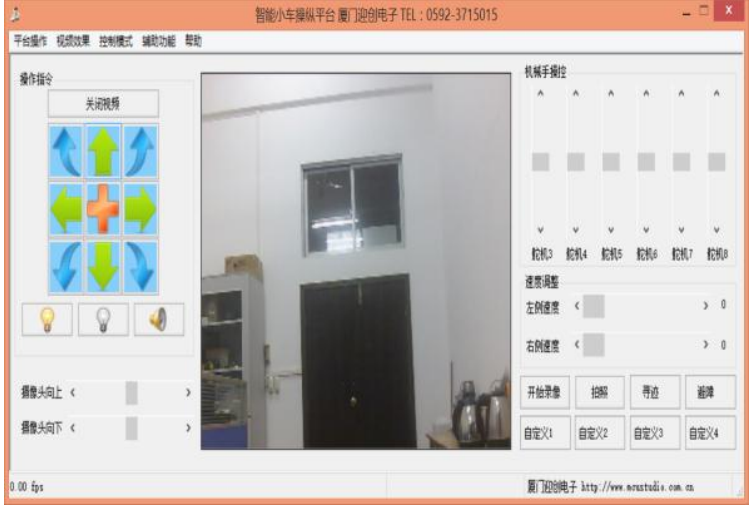

Figure 11. Video images

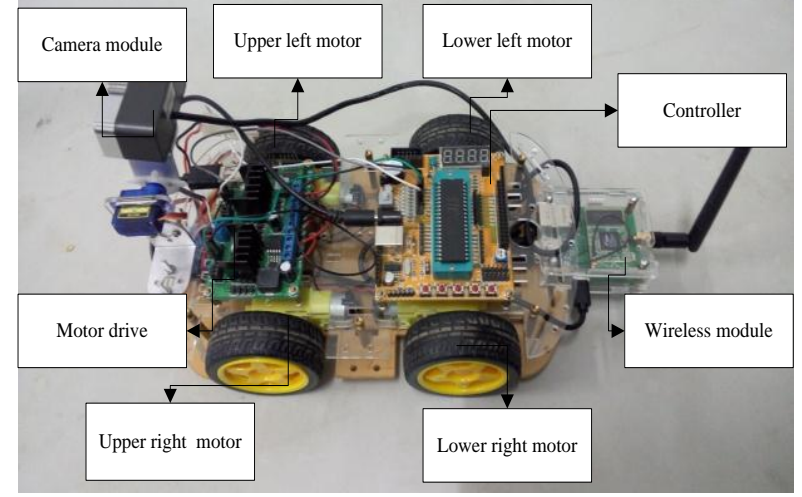

Figure 12. Robot module

\section{Summary}

In this paper, we have explored one approach for solving the question of navigation for blind people: using the four-wheel vehicles as guides. The blind guiding robot is problems that solve blind users navigate safely. We used a STM32 embedded system to design recommendations. We had developed a prototype robot guide with commercially available Blind-guiding Robots. We hope our work will inform and inspire robotics researchers and developers to develop Blind-guiding Robots that can also solve accessibility challenges.

\section{Acknowledgement}

This research was supported by the national natural science foundation of China (Grant No. 61540055), the doctor`s scientific research foundation of Hezhou University (No. HZUBS201506),

\section{References}

[1] Secretary-General, "World demographic trends report", United Nations Economic and Social Council 2009.

[2] "World Report on Disability Estimate based on 2010 population." World Health Organization and World Bank, Geneva 2011.

[3] Mullion, A. Et al. 2009. Indoor positioning and navigation with camera phones. IEEE Pervasive Computing (2009), 22-31.

[4] Ran, L. et al. 2004. Drastic: An integrated indoor/outdoor blind navigation system and service. Proceedings Second IEEE Annual Conference on Pervasive Computing and Communications, PerCom (2004), 23-30.

[5] C. Pyung Hun, P. Sang Rae, J. Je Hyung, P. Sang Hyun. Development of a robot arm assisting people with disabilities at working place using task-oriented design. Rehabilitation Robotics, 2005.

[6] The Lowe's Robot and the Future of Service Work-The New Yorker: http://www. new Yorker. Com/business/currency/lows-robot-future-service-work. Accessed: 2015-10-05.

[7] M.-F. R. Lee and K. H.-E. Lee, "Autonomous target tracking and following mobile robot," Journal of the Chinese Institute of Engineers, Transactions of the Chinese Institute of Engineers, Series A/Chung-kuo Kung Ch'eng Hsuch K'an, 2013, vol. 36, pp. 502-529.

[8] Clarence W. de Silva, "Improved Medical Access through Grama Interactive Environment (IMAGINE)", Department of Mechanical Engineering, The University of British Columbia, Vancouver, Canada, 2013. 
[9] Min-Fan Ricky Lee and Fu-Hsin Steven Chiu "A fuzzy logic navigated service mobile robot" in Proc. International Conference on Fuzzy Theory and Its Application, Taipei, Taiwan, Dec. 6-8, 2013.

[10] Min-Fan Ricky Lee, Nguyen The Hung and Fu-Hsin Steven Chiu "An autonomous mobile robot for indoor security patrol" in Proc. International Conference on Fuzzy Theory and Its Application, Taipei, Taiwan, Dec. 6-8, 2013. 